\title{
The Detection of a Large-mass Planet Around a Ko IV Subgiant With an
}

Almost-circular Orbit

Hiroki Harakawa, Bun'ei Sato, Debra A. Fischer, Shigeru Ida, Masashi Omiya, John A. Johnson, Geoffrey W. Marcy, Eri Toyota, Yasunori Hori, Howard Isaacson, Andrew W. Howard, and Kathryn M. G. Peek

Citation: AIP Conference Proceedings 1158, 245 (2009); doi: 10.1063/1.3215854

View online: http://dx.doi.org/10.1063/1.3215854

View Table of Contents: http://aip.scitation.org/toc/apc/1158/1

Published by the American Institute of Physics 


\title{
The Detection of a Large-mass Planet Around a K0 IV Subgiant With an Almost-circular Orbit
}

\author{
Hiroki Harakawa, Bun'ei Sato, Debra A. Fischer, Shigeru Ida, Masashi \\ Omiya, John A. Johnson, Geoffrey W. Marcy, Eri Toyota, Yasunori Hori, \\ Howard Isaacson, Andrew W. Howard and Kathryn M. G. Peek
}

\begin{abstract}
We report the detection of a new large-mass planet orbiting around a $\mathrm{K} 0 \mathrm{IV}(V=8.26)$ star which has a minimum mass $M_{P} \sin i=10.70 \pm 0.50 M_{\text {Jup }}$ in a $696.0 \pm 2.6$-day orbit. It was detected in precise radial velocity (RV) measurements from Subaru and Keck. The derived orbital parameters, based on a $\chi^{2}$ which minimized by Downhill Simplex algorithm, suggests that these radial velocity variations are consistent with an almost circular planetary orbit and a Mars-like semimajor axis $(e \sim 0.0, a=1.70 \pm 0.03 \mathrm{AU})$. Extra-solar planets that have several times the mass of Jupiter orbiting in periods of hundreds or thousands of days, with very low eccentricities $(e<0.1)$, are rare discoveries. Our detection presents a new sample of these circular orbit massive planets.
\end{abstract}

Keywords: stars: individual:K0 IV, stars: planetary system - techniques: radial velocities - techniques: spectroscopy

PACS: $97.82 . \mathrm{Cp}$

\section{STELLAR CHARACTERISTICS AND ORBITAL SOLUTIONS}

We observed a K0 IV star with $V=8.26, B-V=0.873$, at a distance of $99.4 \mathrm{pc}$, and absolute visual magnitude of $M_{V}=3.27$. We adopted the Downhill Simplex algorithm to reduce $\chi^{2}$, which has multiple parameters (orbital period, time of periastron passage, eccentricity, velocity amplitude and omega - the orientation of the orbit reference to the line of nodes) and obtained the best fit parameters of single Keplerian orbit. Our best fit Keplerian curve is overplotted on the RV data in Figure 1. Before fitting to the RV data, we adopt a "jitter" value of $6 \mathrm{~m} \mathrm{~s}^{-1}$ for our subgiant, and obtained a period of 696.0 days. With the fixed circular orbit we measured a velocity amplitude of $200.0 \mathrm{~ms}^{-1}$. The rms scatter to this fit is $6.60 \mathrm{~ms}^{-1}$ and $\left(\chi_{v}^{2}\right)^{1 / 2}=1.19$ as a reduced $\chi$. The uncertainties in the orbital parameters were estimated by a bootstrap Monte Carlo approach. With a stellar mass of $1.36 M_{\odot}$, we obtained $M \sin i=10.7 M_{J}$ and $a_{p}=1.70 \mathrm{AU}$. The derived stellar characteristics and planet's orbital ele ments are listed in Table 1.

\section{DISCUSSION}

In the core accretion model, massive companions are generally formed with circular orbits because of a protoplanetary disk's circular motion, and our new planet is consistent with this scenario. However, intermediate-period large-mass planets discovered so far tend to be in eccentric orbits. Massive companions are difficult to pertub from their initial orbits due to their large orbital inertia. This suggests an another evolutionary sce-

CP1158, Exoplanets and Disks: Their Formation and Diversity,

Proceedings of the Intemational Conference, edited by T. Usuda, M. Ishii, and M. Tamura

C) 2009 American Institute of Physics 978-0-7354-0695-7/09/\$25.00 


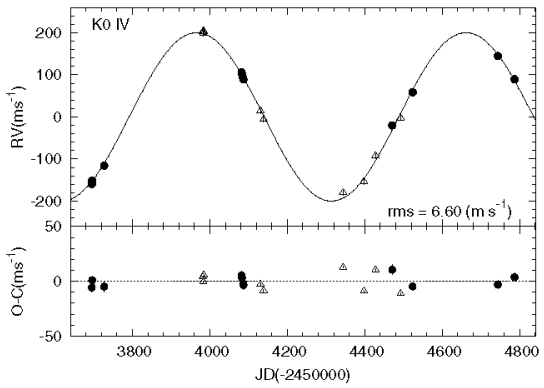

FIGURE 1. Derived RVs for a K0 IV star from Subaru(filled circles) and Keck(opened triangles). With an orbital period of 696.0 days, a velocity gain of $200.0 \mathrm{~ms}^{-1}$, and stellar mass of $1.36 \pm 0.09 M_{\odot}$, the indicated planet mass is $M \sin i=10.7 M_{J}$, the semimajor axis is $1.70 \mathrm{AU}$ and the fixed value of eccentricity $(e=0)$. The rms to the fit is $6.6 \mathrm{~ms}^{-1}$. The offsets of $-58.9 \mathrm{~ms}^{-1}$ and $20.6 \mathrm{~ms}^{-1}$ were applied to the Subaru RVs and the Keck RVs respectively.

TABLE 1. Stellar parameters $(l e f t)$ and derived planet's orbital elements $($ right $)$

\begin{tabular}{lr} 
Stellar Parameter & Value(error) \\
\hline$V$ & 8.26 \\
$M_{V}$ & 3.27 \\
$B-V$ & 0.873 \\
Spectral type & K0 IV(subgiant) \\
Distance(pc) & 99.4 \\
$T_{\text {eff }}(\mathrm{K})$ & $5222(44)$ \\
$\log g$ & $3.84(0.1)$ \\
{$[\mathrm{Fe} / \mathrm{H}]$} & $0.26(0.03)$ \\
$v \sin i\left(\mathrm{kms}^{-1}\right)$ & $0.54(0.5)$ \\
$M_{*}\left(M_{\odot}\right)$ & $1.36(0.09)$ \\
$R_{*}\left(R_{\odot}\right)$ & $2.53(0.47)$ \\
$L_{*}\left(L_{\odot}\right)$ & $4.56(0.13)$ \\
$S_{H K}$ & -0.174 \\
$\log R_{H K}^{\prime}$ & -5.01 \\
\hline
\end{tabular}

\begin{tabular}{lr}
\hline Orbital elements & Value(error) \\
\hline$P$ (days) & $696.0(2.6)$ \\
$T_{c}(\mathrm{JD})$ & $2,453,966.0(2.3)$ \\
Eccentricity & $0($ fixed $)$ \\
$\omega$ & $0($ fixed $)$ \\
$K_{1}\left(\mathrm{~ms}^{-1}\right)$ & $200.0(4.3)$ \\
$a_{1} \sin i\left(10^{-3} \mathrm{AU}\right)$ & $13(0.28)$ \\
$a_{p}(\mathrm{AU})$ & $1.70(0.03)$ \\
$M_{p} \sin i\left(M_{J}\right)$ & $10.7(0.5)$ \\
$N_{\mathrm{obs}}$ & 20 \\
$\mathrm{rms}\left(\mathrm{ms}^{-1}\right)$ & 6.6 \\
$\operatorname{Reduced}\left(\chi_{v}^{2}\right)^{1 / 2}$ & 1.2 \\
\hline
\end{tabular}

nario that could result in eccentric orbits for massive companions (e.g. giant impact[1], disk instability[2]). In Figure 1, some residuals of the single Keplerian fit may have periodic RV variation with a shorter period and smaller amplitude of RV curve than our primary one. However, we could not obtain the significant orbital solutions for multiple Keplerian orbits because of the small number of observations in short term.

\section{REFERENCES}

1. Baraffe, I., Chabrier, G., \& Barman, T. 2008, A\&A, 482, 315

2. Boss, A. P. 1998 , ApJ, 503, 923 